\title{
EXIT Chart Analysis Aided Turbo MUD Designs for the Rank-Deficient Multiple Antenna Assisted OFDM Uplink
}

\author{
Lei Xu, Sheng Chen, Senior Member, IEEE, and Lajos Hanzo, Fellow, IEEE
}

\begin{abstract}
In this paper, the mutual information transfer characteristics of several novel turbo Multiuser Detectors (MUD) employed in Space Division Multiple Access (SDMA) aided Orthogonal Frequency Division Multiplexing (OFDM) systems are investigated with EXtrinsic Information Transfer (EXIT) charts. These novel schemes are the Bayesian, the Soft Interference Cancellation aided Minimum Bit Error Rate (SIC-MBER) and the Reduced-complexity Minimum Bit Error Rate (RMBER) turbo MUDs. In order to increase the effective throughput of the system, a powerful MUD has to be employed in the socalled "rank-deficient" scenario, namely when the number of transmit antennas exceeds that of the receiver antennas. The classic Minimum Mean Square Error (MMSE) solutions exhibit a low complexity. However, they are overwhelmed in rank-deficient scenarios. In these situations powerful non-linear MUDs are required, which are designed in this treatise.
\end{abstract}

Index Terms-EXIT charts, OFDM, MUD, MIMO.

\section{INTRODUCTION}

$\mathbf{S}$ PACE Division Multiple Access (SDMA) aided Orthogonal Frequency Division Multiplexing (OFDM) exhibits a substantially higher achievable system capacity than conventional single-antenna aided systems as a benefit of employing antenna arrays. This is achieved by allowing the users to communicate within the same time-slot and frequency band, differentiating them with the aid of their unique userspecific Channel Impulse Response (CIR)[1,2]. The classic Minimum Mean Square Error (MMSE) aided Multi User Detector (MUD) constitutes an attractive low-complexity design, when the number of uplink Mobile Station (MS) transmitters is lower than the number of Base Station (BS) antennas. However, the MMSE MUD becomes unable to differentiate the users, when the number of MS transmitters exceeds that of the BS's receiver antennas. Nevertheless, the system's performance may be improved by employing the turbo principle [3], resulting in a so-called turbo MUD. Against this background, the novel contribution of this paper is that a range of sophisticated non-linear MUDs are proposed in the context of rank-deficient SDMA OFDM systems and EXIT charts [4] are employed for analyzing their performance.

The rest of this paper is organized as follows. In Section II, our system model is introduced, which will be used in Section III for studying a range of different turbo MUDs. Our simulation results and EXIT chart analysis are presented in Section IV. Finally, we conclude our discourse in Section V.

Manuscript received January 21, 2007; revised May 22, 2007 and July 25, 2007; accepted August 22, 2007. The associate editor coordinating the review of this letter and approving it for publication was D. Hong. The financial support of EPSRC, UK and that of the EU is gratefully acknowledged.

The authors are with the Univ. of Southampton - ECS, Southampton SO17 1BJ UK (e-mail: 1h@ecs.soton.ac.uk).

Digital Object Identifier 10.1109/TWC.2008.070071.

\section{SySTEM MODEL}

The SDMA uplink (UL) transmission structure is depicted in Fig.1. More specifically, each of the $L$ simultaneous MSs employs a convolutional encoder and a single UL transmission antenna, while the BS's UL receiver is equipped with a $P$ element antenna array. As seen in Fig. 1, the complex-valued UL signals, $x_{p}[n, k], p \in 1, \ldots, P$ received from the $P$ element antenna array in the $k$-th subcarrier of the $n$-th OFDM symbol are given by the superposition of the independently faded signals corresponding to the $L$ UL users sharing the same frequency band, which are also contaminated by the Additive White Gaussian Noise (AWGN) encountered at the array elements [2]. The indices $[n, k]$ have been omitted for notational convenience during our forthcoming discourse, yielding [2]:

$$
\mathbf{x}=\mathbf{H s}+\mathbf{n}=\overline{\mathbf{x}}+\mathbf{n},
$$

where $\mathbf{x}$ is the $(P \times 1)$-dimensional vector of the UL received signals, $\mathbf{s}$ is the transmitted $(L \times 1)$-dimensional signal vector generated from the convolutional encoded bits, $\mathbf{n}$ is the $(P \times 1)$-dimensional noise vector and $\overline{\mathbf{x}}$ represents the noiseless component of $\mathbf{x}$. Both the complex-valued UL transmitted signal, $s_{l}$ of the $l$-th user, where $l \in 1, \ldots, L$ and the AWGN process, $n_{p}$, at $p$-th antenna array element, where $p \in 1, \ldots, P$ are assumed to exhibit a zero mean and variances of $\sigma_{l}^{2}$ and $2 \sigma_{n}^{2}$, respectively.

Furthermore, $\mathbf{H}$ is the $(P \times L)$-dimensional Frequency Domain Channel Transfer Function (FDCHTF) matrix constituted by the set of channel transfer factors $H_{p, l}$, which represents the independent, stationary and complex-Gaussian distributed channel gain between the reception array element $p \in 1, \ldots, P$ and the single transmitter antenna of the particular user $l$, having a zero-mean and unit variance.

The a posteriori information $\mathbf{L}^{m, p}\left(s_{l}\right)$ output by the turbo MUD is derived by exploiting both the received signal vector $\mathbf{x}$ and the a priori information $\mathbf{L}^{m, a}\left(s_{l}\right)$ of all the $L \mathrm{UL}$ users, which is the interleaved extrinsic information $\mathbf{L}^{c, e}\left(s_{l}\right)$ generated by the channel decoders for the bits received. Upon subtracting the a priori information $\mathbf{L}^{m, a}\left(s_{l}\right)$ from the a posteriori information $\mathbf{L}^{m, p}\left(s_{l}\right)$, the extrinsic information $\mathbf{L}^{m, e}\left(s_{l}\right)$ output by the MUD is attained. After de-interleaving $\mathbf{L}^{m, e}\left(s_{l}\right)$ is forwarded to a bank of Soft-Input Soft-Output (SISO) channel decoders as the a priori information $\mathbf{L}^{c, a}\left(s_{l}\right)$, in order to generate the a posteriori information $\mathbf{L}^{c, p}\left(s_{l}\right)$ for carrying out the decisions concerning the original source bits. The extrinsic information $\mathbf{L}^{c, e}\left(s_{l}\right)$ output by the $l$-th channel decoders, where $l \in 1, \ldots, L$, is generated by deducting the a priori information $\mathbf{L}^{c, a}\left(s_{l}\right)$ from the a posteriori output $\mathbf{L}^{c, p}\left(s_{l}\right)$ and then interleaved, before it is fed back to the MUD 


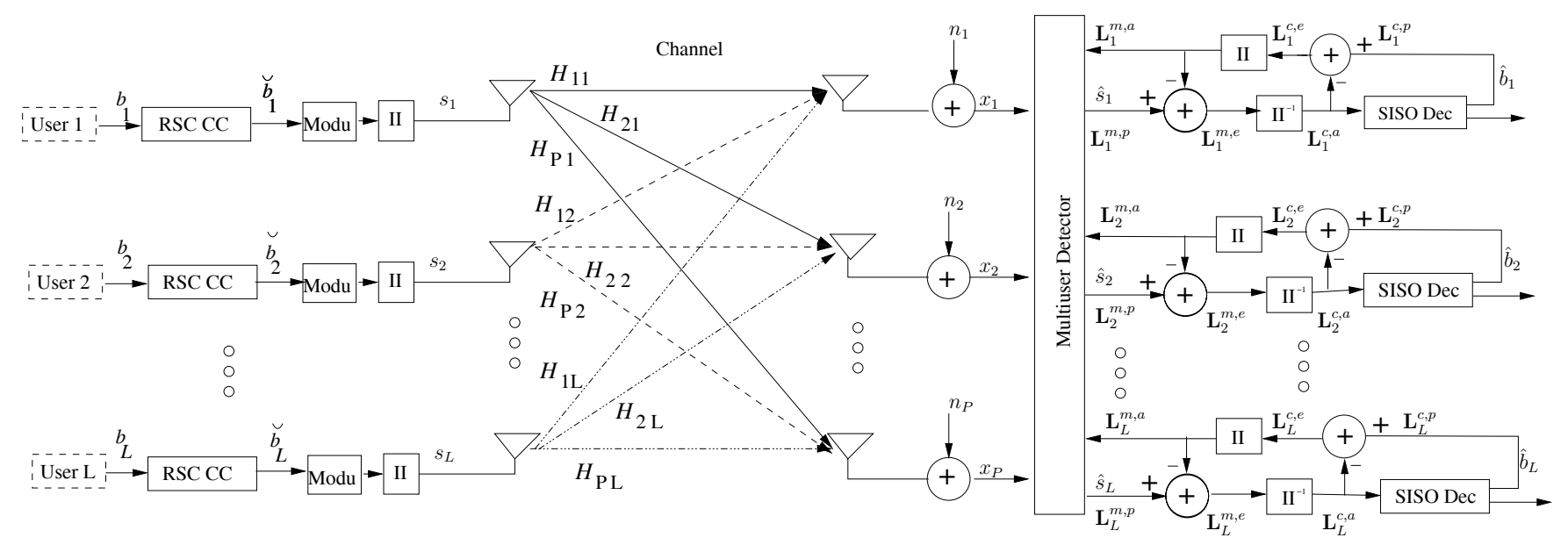

Fig. 1. Schematic of the turbo SDMA OFDM uplink scenario, where each of the $L$ users is equipped with a convolutional channel code and a single transmit antenna, while the BS's receiver is assisted by a $P$-element antenna array followed by iterative processing.

as the a priori information $\mathbf{L}^{m, a}\left(s_{l}\right)$, in order to complete a full iteration. The subscript $l$ of the soft information $\mathbf{L}$ in Fig.1 indicates that it belongs to $s_{l}$.

EXIT charts[4] model the receiver components as blocks that map a sequence of received signal observations and the corresponding a priori information to a new sequence constituting the so-called extrinsic information. It portrays the calculated mutual information of both the extrinsic information $\mathbf{I}_{E}$ and the a priori information $\mathbf{I}_{A}$ as functions of each other in the same coordinate system, as exemplified during our further discouse in SectionIV.

\section{TURBo MUDS}

In this section, we will introduce a novel soft interference cancelation aided MBER turbo MUD, which is referred to as the SIC-MBER solution. For the sake of reducing the inherently high complexity of the SIC-MBER MUD, we additionally introduce a Reduced-complexity MBER (RMBER) MUD, which imposes only a modest degradation of the achievable system performance. Finally, as the ultimate benchmarker, the non-linear Bayesian MUD is introduced.

For M-ary PSK systems supporting $L$ users, the transmitted $L$-user symbol combination may assume $N_{b}=M^{L}$ possible combinations. However, we limit our discusssions here to BPSK $(\mathrm{M}=2)$.

SIC-MBER Turbo MUD: When the turbo MUD using a linear expression to derive the estimates of the transmitted signal employs SIC, the symbol $\hat{s}_{l}^{[i]}$ of the $l$-th user estimated during the $i$-th decoding iteration can be written as follows $[5,6]$ :

$$
\hat{s}_{l}^{[i]}=\bar{s}_{l}^{[i]}+v_{l}^{[i]} \mathbf{w}_{l}^{[i], H} \cdot\left(\mathbf{x}-\mathbf{H} \overline{\mathbf{s}}^{[i]}\right),
$$

where the superscript $[i]$ denotes the $i$-th decoding iteration, while $v_{l}^{[i]}$ is the a priori variance of $\hat{s}_{l}^{[i]}$ given by $[5,6]$ $v_{l}^{[i]}=1-\left|\bar{s}_{l}^{[i]}\right|^{2} . \bar{s}_{l}^{[i]}$ is the $l$-th user's a priori mean value and all the values $\bar{s}_{l}^{[i]}, l=1 \ldots L$ of the $L$ users constitute the mean vector $\overline{\mathbf{s}}^{[i]}$ during the $i$-th decoding iteration. Furthermore, $\mathbf{w}_{l}^{[i], H}$ is the Hermitian of the $l$-th column of the array weight matrix $\mathbf{W}^{[i]}$. The $l$-th user's MBER array weight vector solution during the $i$-th decoding iteration using SIC is defined as:

$$
\mathbf{w}_{l, M B E R}^{[i]}=\arg \min _{\mathbf{w}_{l}^{[i]}} P_{E}\left(\mathbf{w}_{l}^{[i]}\right)=P\left(\operatorname{sgn}\left(s_{l}\right) \hat{s}_{l, R}^{[i]}<0\right) .
$$

where $\hat{s}_{l, R}^{[i]}$ is the real part of the estimated signal $\hat{s}_{l}^{[i]}$.

Given the a priori information concerning the likelihood of all the legitimate $N_{b}=2^{L}$ number of BPSK modulated $L$-user bit sequences $\breve{\mathbf{b}}^{(j)}, j \in 1, \ldots, N_{b}$ and using a similar procedure to that in $[6,7]$, the probability of error $P_{E}$ encountered at the MUD's output regarding the transmitted BPSK-modulated bits $\breve{b}_{l}^{(j)} \in\{ \pm 1\}, j \in 1, \ldots, N_{b}$ of user $l$, which constitutes the resultant MBER cost function, can be written as:

$$
P_{E}\left(\overline{\mathbf{w}}_{l}^{[i]}\right)=\sum_{j=1}^{N_{b}} P^{[i]}\left(s^{(j)}\right) Q\left[\frac{\operatorname{sgn}\left(\breve{b}_{l}^{(j)}\right) \cdot \overline{\hat{s}}_{l, R}^{(j)}}{\sigma_{n}}\right],
$$

where $\overline{\hat{s}}_{l, R}^{(j)}{ }^{[i]}=\Re\left[\bar{s}_{l}^{[i]}+v_{l}^{[i]} \overline{\mathbf{w}}_{l}^{[i], H} \mathbf{H}\left(\breve{\mathbf{b}}^{(j)}-\overline{\mathbf{s}}^{[i]}\right)\right]$, which is the real part of the noiseless center of the l-th user's estimated symbol after SIC, when the $j$-th legitimate BPSK bit sequence is transmitted. Furthermore, $2 \sigma_{n}^{2}$ in Eq.(4) is the variance of the noise, and $\overline{\mathbf{w}}_{l}^{[i]}$ is the unity-norm normalized weight vector during the $i$-th decoding iteration, while $P^{[i]}\left(s^{(j)}\right)$ is the $a$ priori information to be defined more explicitly below.

Provided that the convolutional encoded bits of all $L$ users are independent, the probability $P\left(s^{(j)}\right)$ can be expressed as

$$
P^{[i]}\left(s^{(j)}\right)=\prod_{l=1}^{L} P^{[i]}\left(s_{l}^{(j)}\right), j \in 1, \ldots, N_{b},
$$

where $P^{[i]}\left(s_{l}^{(j)}\right)$ represents the probabilities of either $P^{[i]}\left(s_{l}^{(j)}=1\right)$ or $P^{[i]}\left(s_{l}^{(j)}=0\right)$, depending on the $l$-th user's bit at the $j$-th bit position $j \in 1, \ldots, N_{b}=2^{L}$ in the $L$-user transmitted symbol vector, which is the a priori information provided by the $l$-th user's SISO channel decoder, gleaned from the knowledge of the surrounding bits.

However, in contrast to the closed-form MMSE solutions [5, 8], no closed-form MUD weight solution exists for the MBER 
MUD. Therefore, different techniques based on the Conjucate Gradient (CG) algorithm and on Genetic Algorithms (GAs) have been proposed for approaching the exact MBER solution $[6,7,9]$. The gradient of the BER cost function employing a priori information is given by

$$
\begin{array}{r}
\left.\nabla_{\overline{\mathbf{w}}_{l}^{[i]}} P_{E}\left(\overline{\mathbf{w}}_{l}^{[i]}\right)=\frac{1}{\sqrt{2 \pi} \sigma_{n}} \cdot \sum_{j=1}^{N_{b}} P^{[i]}\left(s^{(j)}\right) e^{\left(-\frac{\left(\overline{\hat{s}}_{l, R}^{(j)}{ }^{[i]}\right)^{2}}{2 \sigma_{n}^{2}}\right)}\right) \\
\cdot \operatorname{sgn}\left(\breve{b}_{l}^{(j)}\right) \cdot\left(\overline{\mathbf{w}}_{l}^{[i]} \overline{\hat{s}}_{l}^{(j)}-v_{l}^{[i]} \mathbf{H}\left(\breve{\mathbf{b}}^{(j)}-\overline{\mathbf{s}}^{[i]}\right)\right),
\end{array}
$$

which may be used by gradient based techniques for generating the weights of the MBER turbo MUD during the $i$-th decoding iteration.

Once the MBER weight matrix has been obtained, the soft a posteriori information of the MBER turbo MUD associated with the $l$-th user during the $i$-th decoding iteration can be written as:

$$
\begin{gathered}
\mathbf{L}^{m, p}\left(s_{l}\right)=\ln \frac{P\left(s_{l}=+1 \mid \hat{s}_{l}^{[i]}\right)}{P\left(s_{l}=-1 \mid \hat{s}_{l}^{[i]}\right)}=\ln \frac{P\left(\hat{s}_{l}^{[i]}, s_{l}=+1\right)}{P\left(\hat{s}_{l}^{i i]}, s_{l}=-1\right)} \\
=\ln \frac{\sum_{\forall j: s_{l}^{(j)}=+1} P^{[i]}\left(s^{(j)}\right) e^{\left(-\frac{\left(\Re\left[\hat{s}_{l}^{[i]}-\bar{s}_{l}^{(j)}[i]\right]\right)^{2}}{2 \sigma_{n}^{2}}\right)}}{\sum_{\forall j: s_{l}^{(j)}=-1} P^{[i]}\left(s^{(j)}\right) e^{\left(-\frac{\left(\Re\left[\hat{s}_{l}^{[i]}-\bar{s}_{l}^{(j)}[i]\right]\right)^{2}}{2 \sigma_{n}^{2}}\right)}} \\
=\ln \frac{\sum_{\forall j: s_{l}^{(j)}=+1} P^{[i]}\left(s^{(j)}\right) e^{\left(-\frac{\left(\Re\left[v_{l}^{[i]} \overline{\mathbf{w}}_{l}^{[i], H}\left(\mathbf{x}-\mathbf{H} \breve{\mathbf{b}}^{(j)}\right]\right)^{2}\right.}{2 \sigma_{n}^{2}}\right)}}{\sum_{\forall j: s_{l}^{(j)}=-1} P^{[i]}\left(s^{(j)}\right) e^{\left(-\frac{\left(\Re\left[v_{l}^{[i]} \overline{\mathbf{w}}_{l}^{[i], H}(\mathbf{x}-\mathbf{H \breve { \mathbf { b } } ( j )}]\right)^{2}\right.}{2 \sigma_{n}^{2}}\right)}},
\end{gathered}
$$

where the exponential term $e^{\left(-\frac{\left(\Re\left[\hat{s}_{l}^{[i]}-\bar{s}_{l}^{(j)}[i]\right]\right)^{2}}{2 \sigma_{n}^{2}}\right)}$ corresponds to the conditional Gaussian PDF of the estimated signal centered at the mean of the noiseless phasor point of the $l$-th user's estimated symbol, when considering the $j$-th legitimate BPSK bit sequence transmitted. Therefore, the summation of all the terms in both the numerator and denominator is constituted by a Gaussian mixture, rather than by a Gaussian distribution. This is the benefit of the MBER MUD in contrast to MMSE solutions. During the first iteration all the probabilities $P^{[i]}\left(s_{l}^{(j)}\right), j \in 1, \ldots, N_{b}, l \in 1, \ldots, L$ are set to $1 / 2$, resulting in $\mathbf{L}^{m, a}\left(s_{l}\right)=0$.

The computational complexity required for the calculation of detection associated with a single transmitted bit can be approximated as $i\left(N_{\text {grad }}\left(N_{b} / 2\right) C^{\nabla P_{E}}+\left(N_{b}-1\right)\right)+N_{b}(2 P(2 L-$ $1)+P(4 L-1)) /(L)$ real-valued multiplications and additions, where $N_{\text {grad }}$ is the number of iterations used by the CG algorithm and $C^{\nabla P_{E}} \approx N_{b}(6 N+2)$ is the compuational complexity required for the calculation of the gradient of Eq. 6.

RMBER Turbo MUD: Observe in Eq.(7) that in the SICMBER turbo MUD, the calculation of the MBER weight vectors is necessary during every iteration and thus it imposes a high complexity. On the other hand, our investigations not included here owing to lack of space revealed that the $a$ priori information $P^{[i]}\left(s^{(j)}\right)$ in Eq.(7) dominates the extrinsic information contribution to the output LLRs. Hence, if the MBER weight matrix is only calculated once during the first iteration and then it is used during the following iterations, this will lead to a significantly reduced complexity at the cost of approximating the LLRs and slightly degrading the achievable system performance. This method is referred to here as the RMBER solution. The computational complexity required for the calculation of detection associated with a single transmitted bit can be given by $i\left(N_{b}-1\right)+N_{\text {grad }}\left(N_{b} / 2\right) N_{b}(6 N+$ $2)+N_{b}(2 P(2 L-1)+P(4 L-1)) /(L)$ real-valued multiplications and additions. The RMBER technique can also be combined with other MUDs, such as the MMSE turbo MUD. This potentially enables us to eliminate the above-mentioned performance loss imposed, which will be illustrated more explicitly in Section IV.

Bayesian Turbo MUD: When the a priori information concerning the likelihood of all the legitimate $N_{b}=2^{L}$ number of BPSK modulated $L$-user bit sequences becomes available during the $i$-th decoding iteration, the joint PDF of the antenna array's output $\mathbf{x}$ and the transmitted BPSK modulated bits $\breve{b}_{l}^{(j)} \in\{ \pm 1\}, j \in 1, \ldots, N_{b}$ of user $l$ at the output of the convolutional encoder can be expressed as the superposition of all the conditional Gaussian PDFs positioned at the legitimate noiseless outputs corresponding to $s_{l}=+1$ and $s_{l}=-1$, multiplied by the $j$-th legitimate signal vector's probabilities, respectively, which can be expressed as:

$$
\begin{aligned}
& P\left(\mathbf{x}, s_{l}=+1\right)=\frac{1}{\sqrt{2 \pi} \sigma_{n}} \sum_{\forall j: s_{l}^{(j)}=+1} P^{[i]}\left(s^{(j)}\right) e^{\left(-\frac{\left(\left\|\mathbf{x}-\overline{\mathbf{x}}_{j}\right\|\right)^{2}}{2 \sigma_{n}^{2}}\right)}, \\
& P\left(\mathbf{x}, s_{l}=-1\right)=\frac{1}{\sqrt{2 \pi} \sigma_{n}} \sum_{\forall j: s_{l}^{(j)}=-1} P^{[i]}\left(s^{(j)}\right) e^{\left(-\frac{\left(\left\|\mathbf{x}-\overline{\mathbf{x}}_{j}\right\|\right)^{2}}{2 \sigma_{n}^{2}}\right)} .
\end{aligned}
$$

The entire set of $N_{b}=2^{L}$ number of legitimate vectors of the $L$ users is partitioned into two subset corresponding to $s_{l}=+1$ and $s_{l}=-1$ according to these two equations. In Eq.(8) and Eq.(9), $\overline{\mathbf{x}}_{j}, j \in 1, \ldots, N_{b}$ constitutes the noiseless received signal vectors, where the Gaussian PDFs seen in Eq.(8) and Eq.(9) are centered.

Based on the above arguments concerning the joint PDF of the received signal vector and the $l$-th user's transmitted bit, we advocate here a novel Bayesian turbo MUD. Let us use the conditional likelihood of the received signal vector as that of the estimated bit decision concerning the $l$ th user's transmitted bit. Then we have:

$$
\begin{array}{r}
\mathbf{L}^{m, p}\left(s_{l}\right)= \\
\ln \frac{P\left(s_{l}=+1 \mid \mathbf{x}\right)}{P\left(s_{l}=-1 \mid \mathbf{x}\right)}=\ln \frac{P\left(\mathbf{x}, s_{l}=+1\right)}{P\left(\mathbf{x}, s_{l}=-1\right)} \\
=\ln \frac{\sum_{\forall j: s_{l}^{(j)}=+1} P^{[i]}\left(s^{(j)}\right) e^{\left(-\frac{\left(\left\|\mathbf{x}-\overline{\mathbf{x}}_{j}\right\|\right)^{2}}{2 \sigma_{n}^{2}}\right)}}{\sum_{\forall j: s_{l}^{(j)}=-1} P^{[i]}\left(s^{(j)}\right) e^{\left(-\frac{\left(\left\|\mathbf{x}-\overline{\mathbf{x}}_{j}\right\|\right)^{2}}{2 \sigma_{n}^{2}}\right)} .} .
\end{array}
$$




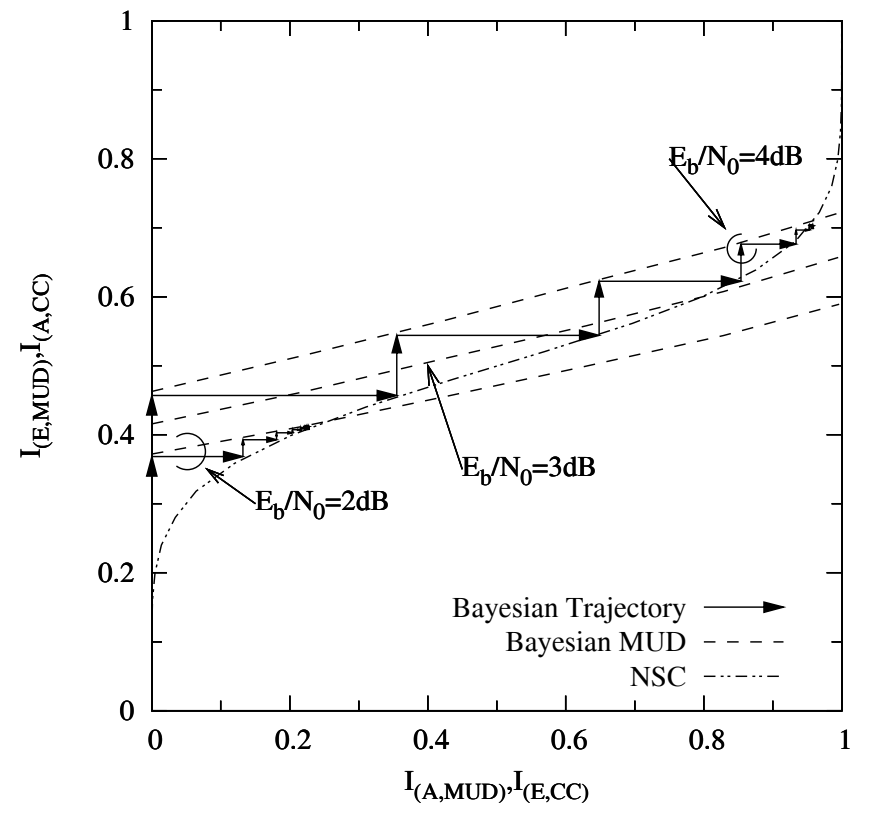

Fig. 2. EXIT chart and simulated detection trajectory of the Bayesian MUD for $P=2$ receiver antennas and $L=6$ users.

This will lead to the maximum-likelihood solution. The computational complexity required for the detection of a single transmitted bit can be shown to be given as $i\left(2 N_{b}+6 N_{b} P / L-\right.$ $1)+N_{b}(2 P(2 L-1)+P(4 L-1)) /(L)$ real-valued multiplications and additions.

\section{Simulation RESUlts}

Since the individual users' signals interfere with each other and influence each other's a priori mutual information $I_{A}$ and extrinsic mutual information contributions $I_{E}$, an $L$ dimensional EXIT chart would be required. In order to circumvent this problem and hence allow us to plot the EXIT curves for a multi-user communication system in a two-dimensional, rather than $L$-dimensional plane, the average of all the users' mutual information is required. Provided that the average of each Channel Impulse Response (CIR) tap as well as each user's bit energy $\left(E_{b}\right)$ to noise power spectral density $\left(N_{0}\right)$ ratio is similar, then these assumptions may be judiciously exploited for the sake of simplifying the $L$-user EXIT chart analysis. Perfect Channel State Information (CSI) is assumed in our simulations. The channel impulse response used is 3path SWATM[2], and the OFDM has 128 subcarriers. The code rate is $1 / 2$.

EXIT Chart Trajectories of the Proposed MUDs: In Fig.2, we plot both the EXIT charts and the simulated detection trajectories of the Bayesian turbo receiver supporting $L=6$ users $^{1}$ with the aid of $P=2$ receiver antenna elements. We denote the a priori information and extrinsic information of the MUD by $\mathbf{I}_{(\mathbf{A}, \mathbf{M U D})}$ and $\mathbf{I}_{(\mathbf{E}, \mathbf{M U D})}$, respectively, while the corresponding quantities of the channel decoder by $\mathbf{I}_{(\mathbf{A}, \mathbf{C C})}$ and $\mathbf{I}_{(\mathbf{E}, \mathbf{C C})}$, respectively. It is shown

\footnotetext{
${ }^{1}$ Conventional SDMA MUD schemes can only support two users with the aid of two receiver antennas. The novelty of the proposed solution is that it is capable of supporting up to three times more users in this so-called rank-deficient scenario.
}

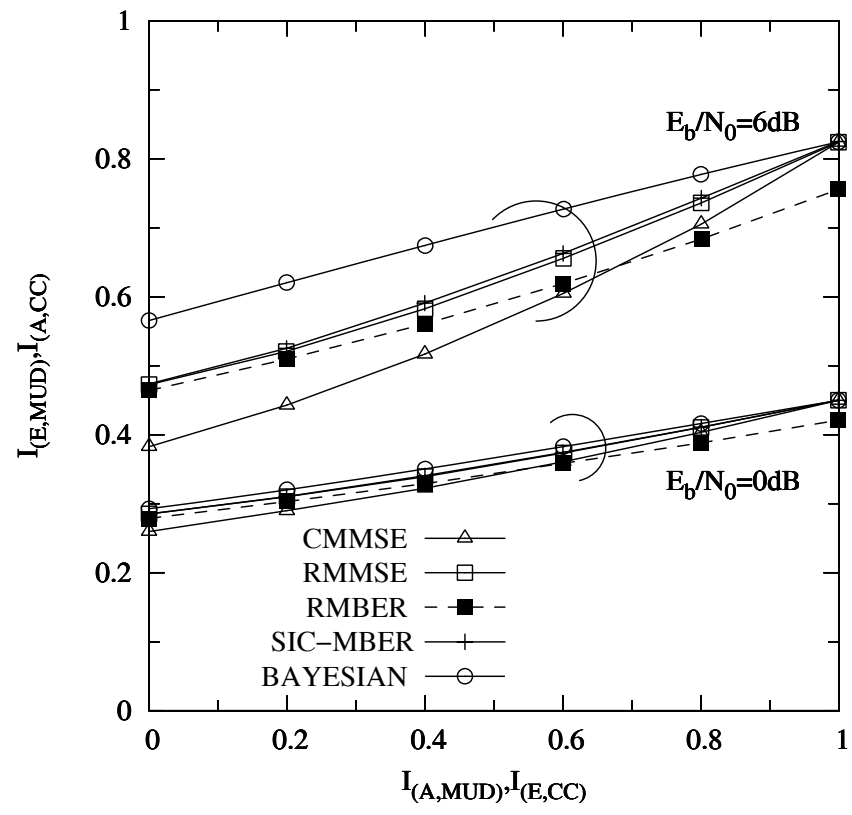

Fig. 3. EXIT charts of different turbo MUDs for $P=2$ receiver antennas and $L=6$ users.

that the simulated decoding trajectory evolves within the open detection tunnel between the EXIT curves of the MUD and the channel decoder, until it reaches the intersection of the curves. Since the simulated detection trajectories closely follow the EXIT curves of the receiver components, the validity of EXIT chart analysis is demonstrated.

When the average $E_{b} / N_{0}$ value is insufficiently high, as seen for $E_{b} / N_{0}=2 d B$ in Fig. 2, the trajectory becomes unable to reach the top-right corner, since it is curtailed after three iterations. By contrast, at $E_{b} / N_{0}=4 d B$, the decoding trajectory arrives at the top-right corner, indicating that the MUD is indeed capable of approaching the single-user bound.

Comparison of Different Turbo MUDs: In Fig.3, we plot the EXIT curves of different turbo MUD schemes including the Complex-valued MMSE (CMMSE) [8] and Real-valued MMSE (RMMSE) [10] schemes as benchmarkers, as well as the proposed SIC-MBER, RMBER and Bayesian detectors, when supporting $L=6$ users at both $E_{b} / N_{0}=0 d B$ and $6 d B$. At the abscissa value of unity, all the EXIT curves of the different MUDs recorded at a given SNR value converge to the same point, coinciding with that recorded for a single user, except for the RMBER turbo MUD. This reveals that the multiple access interference (MAI) is virtually eliminated by these MUDs, provided that perfect a priori information is available. For the RMBER turbo MUD, the approximation described before prevents us from completely eliminating the MAI even for $\mathbf{L}^{m, a}\left(s_{l}\right)=1$, and this degrades the attainable performance.

The Bayesian turbo MUD exhibits the highest starting point at the abscissa of zero in Fig.3, followed by the SICMBER and RMBER schemes, the RMMSE and finally the CMMSE MUD. Hence the Bayesian MUD has the lowest SNR convergence threshold, followed by the SIC-MBER, the RMMSE and the CMMSE MUD. It is also notable that the distances between the starting points of the EXIT curves seen 


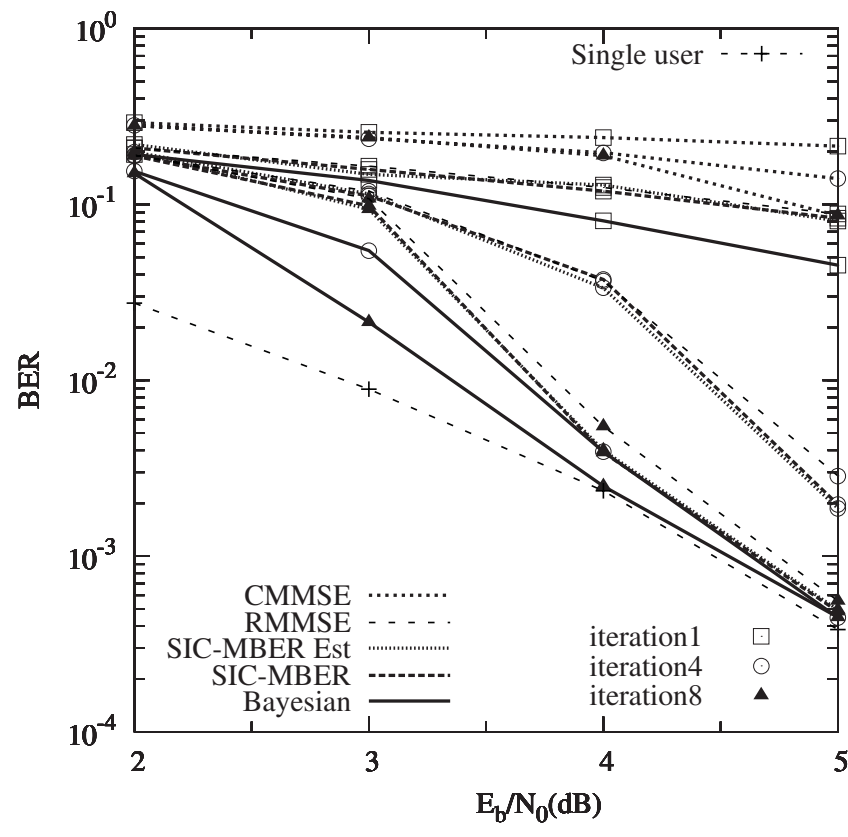

Fig. 4. BER performance of the different MUDs for $P=2$ receiver antennas and $L=6$ users, where the curve denoted as "SIC-MBER Est" is the estimated BER performance of the SIC-MBER MUD.

in Fig. 3 for these different schemes tend to increase, as the SNR becomes higher. This is because when the signal is heavily contaminated by the noise at a low SNR, all the turbo schemes failed to provide a high confidence concerning the detected soft output.

Fig.4 portrays the attainable BER performance of all the MUDs parameterized by the SNR, except for the RMBER detector, which confirms the predictions of our EXIT chart analysis. It is clearly seen in Fig.4 that when the SNR is higher than a certain threshold and the number of iterations is sufficiently high, the achievable BER of the proposed MUDs approaches the single-user-bound.

EXIT Chart Based BER Estimation: When the Gaussian distribution assumption is applied to the PDF of LLRs, the extrinsic mutual information $\mathbf{I}_{E}$ or a priori mutual information $\mathbf{I}_{A}$ will be a function of a single parameter, namely that of the LLR variance $\sigma_{L L R}^{2}[4]$,

$\mathbf{I}\left(\sigma_{L L R}\right)=1-\int_{-\infty}^{\infty} \frac{e^{-\left(x-\sigma_{L L R}^{2} / 2\right)^{2} / 2 \sigma_{L L R}^{2}}}{\sqrt{2 \pi} \sigma_{L L R}} \cdot \log _{2}\left(1+e^{-x}\right) d x$.

Let us assume that both the extrinsic information $\mathbf{L}^{c, e}\left(s_{l}\right)$ and the a priori information $\mathbf{L}^{c, a}\left(s_{l}\right)$ is Gaussian distributed with a variance of $\sigma_{e}^{2}$ or $\sigma_{a}^{2}$, respectively, and that they are perfectly independent from each other. Either $\sigma_{e}^{2}$ or $\sigma_{a}^{2}$ can be derived by substituting $\mathbf{L}^{c, e}\left(s_{l}\right)$ or $\mathbf{L}^{c, a}\left(s_{l}\right)$ into the inverse function of Eq.(11), respectively. Thus the decoder's soft output $\mathbf{L}^{c, p}\left(s_{l}\right)$ is also Gaussian distributed, having a variance of $\sigma_{p}^{2}=\sigma_{e}^{2}+\sigma_{a}^{2}$ and a mean of $\mu=\sigma_{p}^{2} / 2$. Then the channel coded bit error probability can be written as [4]

$$
P_{b} \approx Q\left[\frac{\sigma_{p}}{2}\right] \text {. }
$$

In Fig.4, we also compare the estimated channel coded BER results obtained from the EXIT chart to the simulation

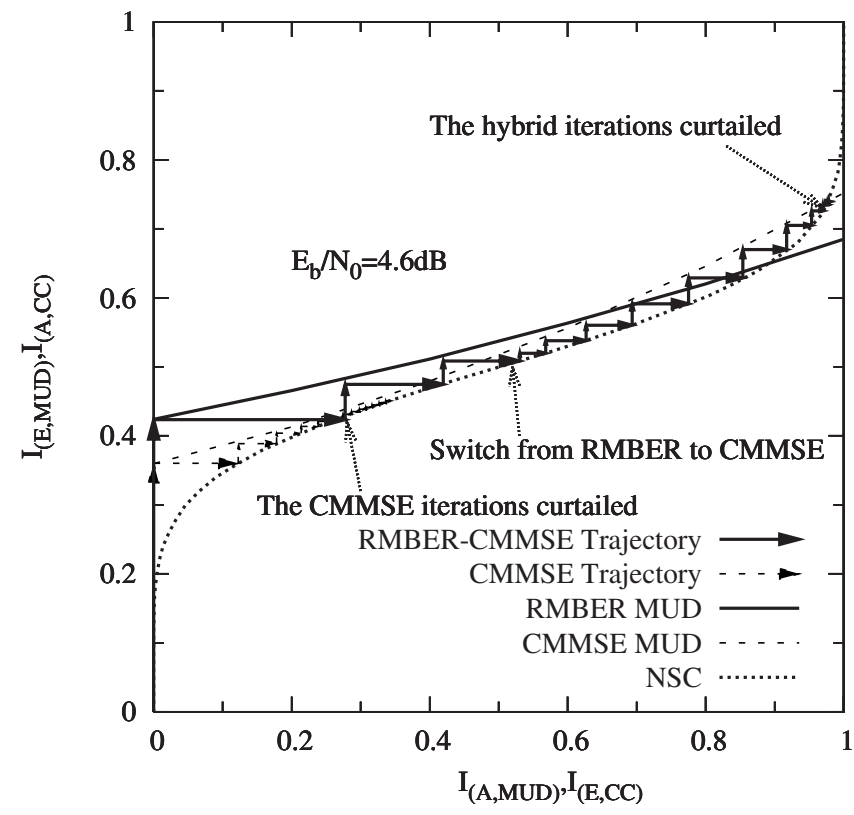

Fig. 5. EXIT chart of the hybrid MUD combining the RMBER and the CMMSE MUDs, which employs the RMBER MUD for the initial three iterations and then invokes the CMMSE MUD for $P=2$ receiver antennas and $L=6$ users.

results characterizing the SIC-MBER turbo MUD. The figure illustrates that as expected, the BER estimation employing the Gaussian mutual information approximation is sufficiently accurate.

Adaptive Combination of Different Turbo MUDs: If we use the RMBER MUD during a few initial iterations and subsequently employ the CMMSE or the RMMSE criterion for further processing the a priori information, the MUD would be able to avoid any performance degradation and inherit the joint advantages of the combined MUD, since as seen in Fig.3, the RMBER detector requires a lower initial SNR and attains a more rapid convergence rate than the CMMSE MUD. In comparison to the full-complexity SICMBER MUD characterized in Fig.3, this scheme achieves a similar performance at a significantly lower complexity.

Fig.5 characterizes the above-mentioned hybrid MUD, which employs the RMBER MUD during the initial three iterations and then invokes the CMMSE scheme, when supporting $L=6$ users with the aid of $P=2$ receiver antenna elements at $E_{b} / N_{0}=4.6 d B$. The EXIT curves of the RMBER and the CMMSE MUDs along with their simulated trajectories are shown in Fig.5. We can see that the CMMSE trajectory gets trapped at the intersection between the EXIT curves of the channel decoder and the MUD. By contrast, the MUD combining the RMBER and the CMMSE MUD exhibits a marginally open tunnel between the EXIT curves of the hybrid MUD and the channel decoder, hence it becomes capable of approaching the single-user performance.

The Number of Users Supported: Observe in Fig.6 that at a certain SNR, the EXIT curve of the MUD moves downwards, when the number of users $L$ is increased, potentially closing the open convergence tunnel. This limits the maximum number of users supported by the system at this SNR.

More explicitly, Fig.6 plots the EXIT curves of the Bayesian 


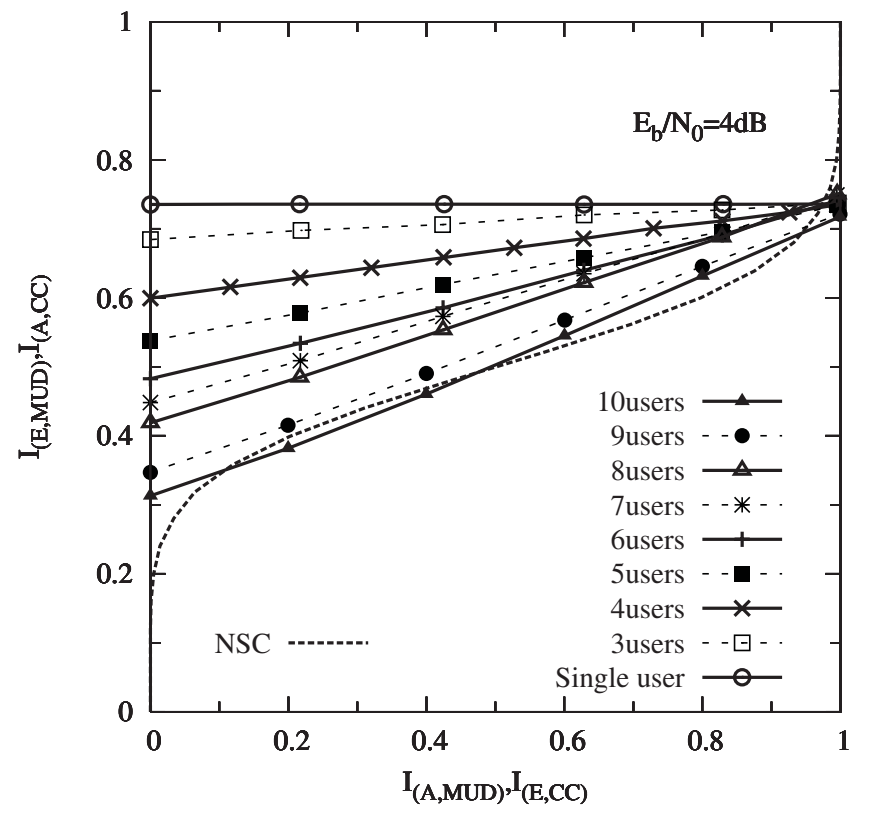

Fig. 6. EXIT characteristics of the Bayesian MUD for supporting different number of users at $E_{b} / N_{0}=4 d B$, when employing $P=2$ receiver antennas.

MUD, when supporting different number of users at $E_{b} / N_{0}=$ $4 d B$, along with that of the previously used NSC code. It is seen that the Bayesian MUD is capable of supporting $L=9$ users with the aid of $P=2$ receiver antennas, while maintaining an open tunnel between the EXIT curves of the MUD and the channel decoder. We can also see in Fig.6 that all the curves corresponding to the different numbers of users converge to the same point around the ordinate value of $I_{(E, M U D)} \approx 0.74$ at the abscissa of $I_{(A, M U D)}=1$, again, attaining a near-single-user performance. It is also worth noting in Fig. 6 that the point of perfect convergence at $(1,1)$ is not reached at $E_{b} / N_{0}=4 d B$. This is because the BER of the single-user system is excessive at this SNR. Naturally, when the SNR is high and the number of users is sufficiently low, the point of $(1,1)$ can indeed be reached.

\section{Conclusion}

In conclusion, a turbo Bayesian and a turbo SIC-MBER MUD was proposed, both of which are capable of outperform- ing the CMMSE and RMMSE detection aided turbo MUDs. For the sake of reducing the complexity of the SIC-MBER algorithm, the RMBER scheme was proposed at the cost of a modest performance degradation. In order to mitigate this degradation, a hybrid MUD was also proposed, which activated the RMBER MUD during the first three iterations, followed by a specific number of CMMSE MUD iterations required for avoiding any performance deterioration. Although the output of the proposed turbo Bayesian or SIC-MBER MUD is potentially non-Gaussian, the conventional singleuser EXIT-chart analysis remains applicable in the MUD-aided scenario, adequately predicting the convergence behavior of these turbo MUDs. We also demonstrated the accuracy of the EXIT charts in predicting the achievable BER performance of the MUDs as well as in characterizing their convergence behavior.

\section{REFERENCES}

[1] P. Vandenameele, L. van Der Perre, and M. Engels, Space Division Multiple Access for Wireless Local Area Networks. Boston: Kluwer Academic Publishers, 2001.

[2] L. Hanzo, M. Münster, B. J. Choi, and T. Keller, OFDM and MCCDMA. West Sussex, England: John Wiley and IEEE Press, 2003.

[3] C. Berrou, A. Glavieux, and P. Thitimajshima, "Near-optimum errorcorrecting coding and decoding: Turbo codes," IEEE Trans. Commun., vol. 44, pp. 1261-1271, Oct. 1996.

[4] S. ten Brink, "Convergence of iterative decoding," IEE Electron. Lett., vol. 35, no. 13, pp. 1117-1118, June 1999.

[5] M. Tuchler, A. C. Singer, and R. Koetter, "Minimum mean squared error equalization using a priori information," IEEE Trans. Signal Processing, vol. 50, pp. 673-683, Mar. 2002.

[6] M. Y. Alias, S. Chen, and L. Hanzo, "Multiple-antenna-aided OFDM employing genetic-algorithm-assisted minimum bit error rate multiuser detection," IEEE Trans. Veh. Technol., vol. 54, pp. 1713-1721, Sept. 2005.

[7] M. Y. Alias, A. K. Samingan, S. Chen, and L. Hanzo, "Multiple antenna aided OFDM employing minimum bit error rate multiuser detection," IEE Electron. Lett., vol. 39, no. 24, pp. 1769-1770, 27 Nov. 2003.

[8] H. V. Poor, "Iterative multiuser detection," IEEE Signal Processing Mag., pp. 81-88, Jan. 2004.

[9] S. Chen, A. K. Samingan, B. Mulgrew, and L. Hanzo, "Adaptive minimum-BER linear multiuser detection for DS-CDMA signals in multipath channels," IEEE Trans. Signal Processing, vol. 49, no. 6, pp. 1240-1247, June 2001.

[10] W. R. Schober and L. Lampe, "On suboptimum receivers for DS-CDMA with BPSK modulation,” Signal Processing, vol. 85, no. 6, pp. 1149 1163, June 2005. 